\title{
Apendicitis aguda en paciente adulto con rotación intestinal anormal y defecto cardiovascular: presentación de caso y revisión temática
}

\author{
Acute appendicitis in an adult patient with abnormal intestinal rotation and \\ a cardiovascular defect: case presentation and literature review
}

\author{
Andrea Nathalia Villabona1, Laura Isabel Sorzano1, Héctor Mauricio Rodríguez, \\ Luis Alfredo Espinosa', Sergio Alberto Villabona²
}

Médico, Grupo de Investigación de Urgencias y Trauma, Universidad Autónoma de Bucaramanga, Bucaramanga, Colombia

2 Médico, especialista en Medicina de Urgencias, Departamento de Urgencias, Fundación Oftalmológica de Santander-Clínica Carlos Ardila Lulle, Grupo de Investigación de Urgencias y Trauma, Universidad Autónoma de Bucaramanga, Bucaramanga, Colombia

\section{Resumen}

La apendicitis aguda es la enfermedad quirúrgica que se presenta con mayor frecuencia en los servicios de urgencias. Su diagnóstico es predominantemente clínico; alrededor de dos terceras partes de los pacientes con apendicitis presentan un dolor típico localizado en el cuadrante inferior derecho. Sin embargo, hay manifestaciones atípicas, infrecuentes, como el dolor en el cuadrante inferior izquierdo.

Se hace pues, importante conocer la existencia de ciertas enfermedades que puedan explicar estos síntomas para así tenerlas en cuenta en el momento de abordar un paciente con dolor abdominal. Estas son la rotación intestinal anormal y, con mayor frecuencia, el situs inversus, que explican el dolor abdominal secundario a la apendicitis en una localización diferente a la clásica para hacer el diagnóstico y dar tratamiento oportuno, con el fin de prevenir complicaciones. Además, los estudios complementarios son importantes en estos pacientes para descartar otras alteraciones o malformaciones, como defectos cardiovasculares, los que se han relacionado con anomalías en la formación y la rotación gastrointestinal en $27 \%$ de los casos, aproximadamente.

Se presenta el caso de hombre joven, con un cuadro clínico de dolor abdominal que se inició en el epigastrio y después se localizó en el cuadrante inferior izquierdo, acompañado de signos de irritación peritoneal y alteración de los exámenes paraclínicos. Los estudios de imagen confirmaron la presencia de rotación intestinal anormal y apendicitis aguda izquierda. Durante el seguimiento del paciente, se practicó una ecocardiografía transesofágica por dolor torácico, la cual demostró insuficiencia valvular aórtica secundaria a valvulopatía aórtica bivalva. Palabras clave: apendicitis; diagnóstico; apendicectomía; laparotomía; situs inversus; cardiopatías congénitas.

\footnotetext{
Abstract

Acute appendicitis is the most frequent surgical pathology at the emergency departments and its diagnosis is predominantly clinical. Around 2/3 of the patients with this pathology present typical pain, located in the right lower quadrant. However, abdominal pain in the left lower quadrant secondary to acute appendicitis is of

Fecha de recibido: 12/06/2017 - Fecha aceptación: 16/10/2017

Correspondencia: Andrea Nathalia Villabona, MD, Calle 157 № 14-55, Universidad Autónoma de Bucaramanga, Campus El Bosque, Floridablanca, Colombia /Teléfonos: 6991347 y (318) 782-5671

Correo electrónico: avillabona88@unab.edu.co

Citar como:. Villabona AN, Sorzano LI, Rodríguez HM, Espinosa LA, Villabona SA. Apendicitis aguda en paciente adulto con rotación intestinal anormal y defecto cardiovascular: presentación de caso y revisión temática. Rev Colomb Cir. 2018;33:220. https://doi. org/10.30944/20117582.65
} 
infrequent presentation. It is important to know the existence of certain pathologies that can explain the cause for this entity, to take them into account when examining a patient. These are the intestinal malrotation and, most frequently, situs inversus. Both of them explain the abdominal pain secondary to appendicitis in a different location from the classic presentation, in order to make an accurate diagnosis and treatment so complications can be prevented. Additionally, it is important to perform complementary studies in these patients to discard other alterations or malformations, such as cardiovascular defects, because they are related to defects in the gastrointestinal formation and rotation in approximately $27 \%$ of the cases.

We present the case of a young male, with abdominal pain that begins in the epigastrium with posterior localization in the left lower quadrant. He presents signs of peritoneal irritation and abnormal laboratory tests. Imaging studies are performed and confirm the presence of left sided acute appendicitis and intestinal malrotation. During the patient's follow-up, a transesophageal echocardiography was performed due to chest pain, which shows aortic valve insufficiency secondary to aortic bivalve valvulopathy.

Key words: appendicitis; diagnosis; appendectomy; laparotomy; situs inversus; heart defects, congenital.

\section{Introducción}

La apendicitis aguda es una de las enfermedades intraabdominales más frecuentes que requieren manejo quirúrgico ${ }^{\mathrm{I}}$. Su incidencia es, aproximadamente, de I,33 por cada I.Ooo hombres y de 0,99 por cada I.00o mujeres ${ }^{2}$. La sintomatología se caracteriza por dolor abdominal localizado en el cuadrante inferior derecho, principalmente asociado con signos de irritación peritoneal ${ }^{3,4}$. Durante el transcurso de la enfermedad, se pueden presentar signos y síntomas inespecíficos, como fiebre y anorexia ${ }^{3}$, los cuales se encuentran en 50 a $60 \%$ de los pacientes ${ }^{5}$.

Una tercera parte de los pacientes con apendicitis aguda presenta dolor abdominal localizado en una región diferente al cuadrante inferior derecho, encontrándose raramente dolor en el cuadrante inferior izquierdo ${ }^{6}$. La apendicitis aguda ubicada en el cuadrante inferior izquierdo, se puede presentar en el caso de un apéndice largo en un ciego derecho o relacionarse con defectos congénitos, como rotación intestinal anormal o situs inversus ${ }^{1,7}$. El situs inversus es poco común y se produce por un gen autosómico recesivo, con una incidencia que varía entre I por cada 5.000 y I por cada I0.000 nacidos vivos; puede ser total o parcial, dependiendo de si compromete la cavidad abdominal y la torácica, o solo una de las dos ${ }^{\mathrm{I}}$.

La rotación intestinal anormal es un defecto congénito que se presenta en la rotación y fijación del intestino ${ }^{8}$. Puede afectar entre 0,03 y I \% de la población ${ }^{1,9}$. Su diagnóstico en el adulto se hace, aproximadamente, en la mitad de la población que la padece. En los adultos, es común que sea asintomática ${ }^{\mathrm{IO}}$. Sin embargo, su diagnóstico se hace principalmente en el servicio de urgencias, por presencia de dolor abdominal ${ }^{10-12}$.

En algunas ocasiones, la rotación intestinal anormal puede asociarse con defectos cardiovasculares. En un estudio en Holanda, se encontró que dichos defectos congénitos se presentaban en el $27,1 \%$ de la población pediátrica con rotación intestinal anormal, y correspondían a defectos mayores en el 48,I \% de los casos y, a defectos menores, en el 5I,9 \% ${ }^{13}$. Este hecho representa un reto para el tratamiento de estos pacientes, puesto que la tasa de mortalidad después del manejo quirúrgico es elevada en quienes presentan rotación intestinal anormal con comorbilidad cardiaca ${ }^{13}$.

Debido a la carencia de experiencia y de información sobre esta condición a nivel nacional, y a su escasa sospecha diagnóstica ante un paciente con dolor abdominal, se presenta este reporte de caso.

\section{Caso clínico}

Se trata de un paciente de 23 años de edad de sexo masculino, sin antecedentes patológicos, quirúrgicos o familiares de importancia. 
Consultó al servicio de urgencias por un cuadro clínico de 12 horas de evolución, caracterizado por dolor abdominal epigástrico, de tipo retortijón, progresivo y muy intenso, que no se atenuaba en ninguna posición y que se había ido localizando en el flanco y la fosa iliaca izquierdo, asociado con hiporexia y múltiples episodios eméticos de contenido alimentario.

En el examen físico se encontró un paciente en regulares condiciones generales, con estabilidad hemodinámica, deshidratación leve y posición antálgica. Presentaba signos de irritación peritoneal, defensa y dolor a la palpación generalizada del abdomen, con predominio en fosa ilíaca izquierda, signo de Blumberg dudoso, y signos de Rovsing y del talón izquierdo positivos. Sus signos vitales eran: tensión arterial de $132 / 76$ $\mathrm{mm} \mathrm{Hg}$, frecuencia cardiaca de 77 latidos por minuto, frecuencia respiratoria de 20 por minuto, temperatura de $37^{\circ} \mathrm{C}$ y saturación de oxígeno de $98 \%$. El resto del examen físico estuvo dentro de parámetros normales.

En los exámenes de laboratorio se encontró leucocitosis $\left(2 \mathrm{I} .500 / \mathrm{mm}^{3}\right)$, neutrofilia $(86 \%)$ y proteína C reactiva de $16,3 \mathrm{mg} / \mathrm{L}$. La ecografía total de abdomen estuvo dentro de parámetros normales. En la tomografía computadorizada de abdomen (figura I y figura 2) se observó que el bulbo y la segunda porción del duodeno tenían un recorrido habitual, pero la tercera y la cuarta porción no cruzaban la línea media, identificándose asas intestinales delgadas (yeyuno e íleon) en el hemiabdomen derecho. La válvula ileocecal se encontraba en la fosa ilíaca izquierda, y el apéndice cecal estaba aumentado de tamaño e hipodenso, sin captación del medio de contraste, con un diámetro aproximado de $7,3 \mathrm{~mm}$ en la punta, 7,5 en la base y 7,2 en el tercio medio. Por lo anterior, se consideró el diagnóstico de rotación intestinal anormal, con válvula ileocecal en la fosa iliaca izquierda y apendicitis aguda izquierda.

El paciente fue sometido a cirugía de urgencia. Mediante una incisión mediana por planos hasta la cavidad, se localizó el apéndice, el cual se encontraba significativamente aumentado de tamaño y edematoso; se procedió a sección meso-
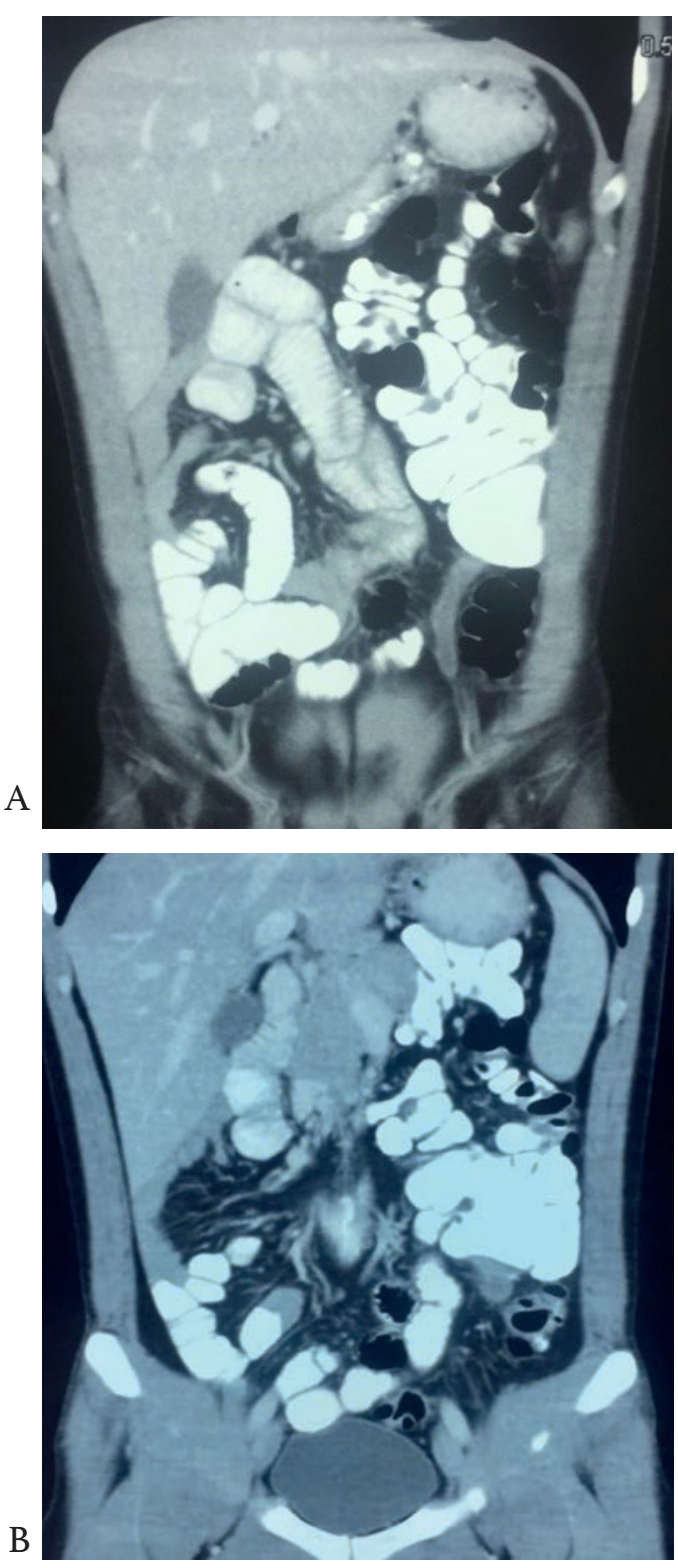

Figura 1 A y B. Tomografía axial con contraste de abdomen (vista coronal): (A) se evidencian asas intestinales delgadas (yeyuno e íleon) en el hemiabdomen derecho. La válvula ileocecal se encuentra en la fosa iliaca izquierda, el apéndice cecal está aumentado de tamaño e hipodenso, sin captación del medio de contraste, con un diámetro aproximado de 7,3 mm en la punta, 7,5 mm en la base y 7,2 mm en el tercio medio. (B) Hígado, bazo y estómago en su sitio. Asas del intestino delgado y ángulo de Treitz a la derecha. Colon a la izquierda. Base del muñón del apéndice grueso, con ausencia de captación del medio de contraste. 

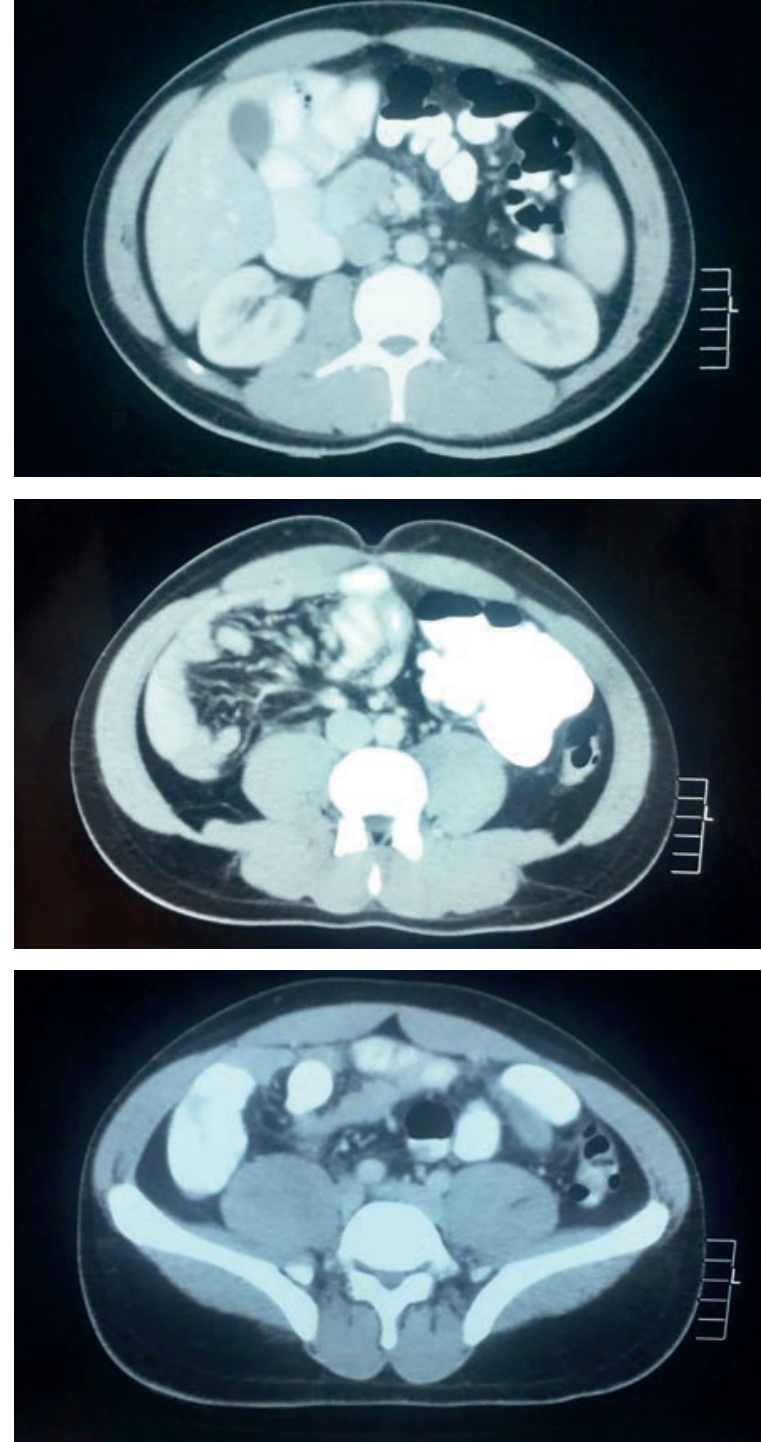

Figura 2. Tomografía axial con contraste de abdomen (vista transversal): se evidencian hígado y bazo en posición habitual. Asas intestinales delgadas y ángulo de Treitz en hemiabdomen derecho. El colon se ubica en el hemiabdomen izquierdo. No se aprecia el apéndice.

apendicular y por la base, y posteriormente, se cerró por planos. No se presentaron complicaciones durante el procedimiento. La evolución clínica fue satisfactoria y se dio egreso hospitalario después de 48 horas de observación.

Durante el seguimiento, tres meses después, el paciente presentaba dolor torácico ocasional, esporádico y de intensidad moderada, razón por la cual se practicó una ecocardiografía transesofágica y se observó insuficiencia valvular aórtica moderada secundaria a una válvula aórtica bivalva sin estenosis (figura 3 ).
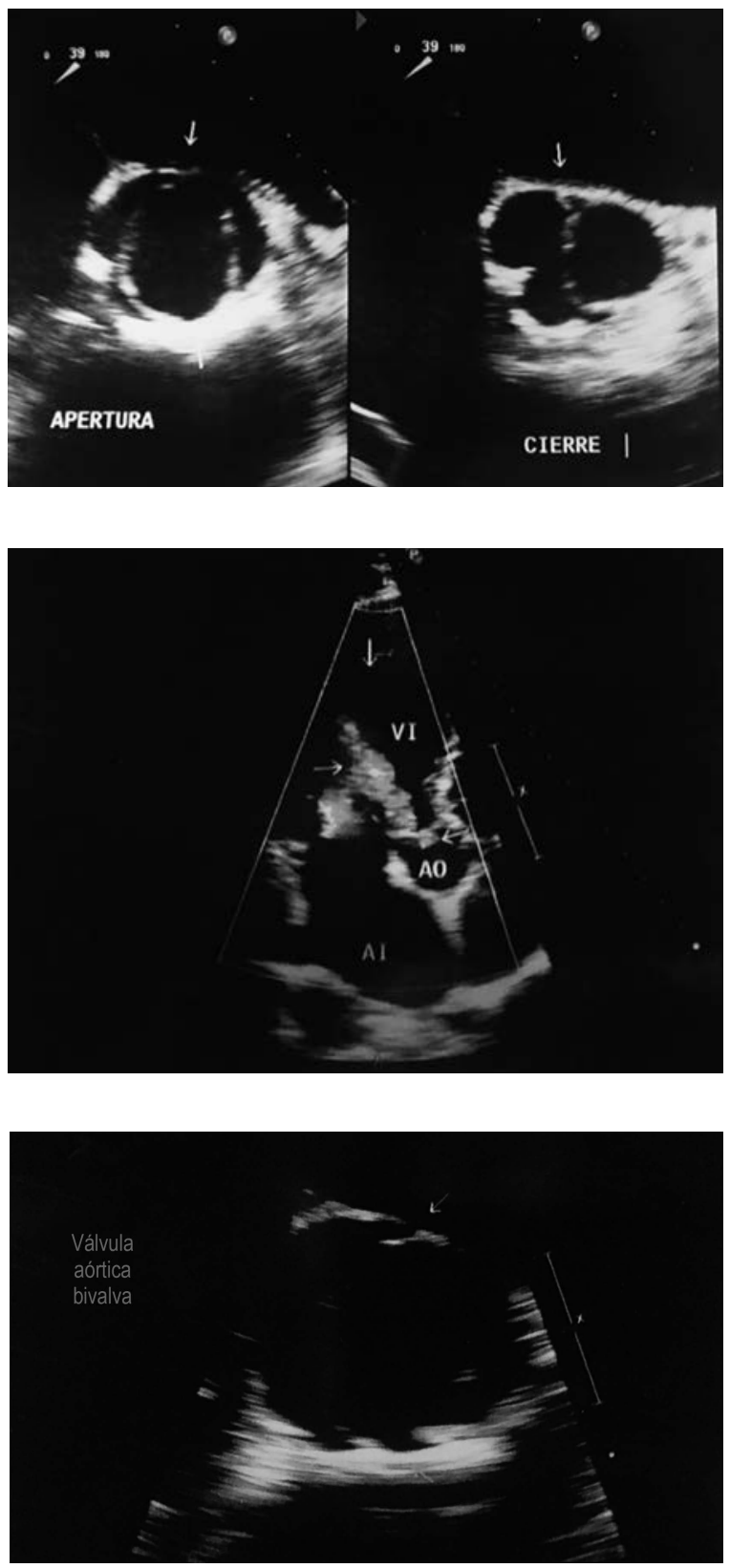

Figura 3. Ecocardiograma transesofágico: se evidencia insuficiencia valvular aórtica moderada secundaria a una válvula aórtica bivalva sin estenosis. 


\section{Discusión}

La apendicitis aguda es una de las enfermedades intraabdominales más frecuentes y la principal urgencia quirúrgica abdominal ${ }^{1,14}$. Usualmente, se presenta como un dolor sordo epigástrico o periumbilical, acompañado de náuseas o emesis, fiebre e hiporexia. Horas después, se irradia y se localiza en el cuadrante inferior derecho ${ }^{4,15}$. No obstante, su presentación en el cuadrante inferior izquierdo es infrecuente ${ }^{6}$, y puede relacionarse con dos defectos congénitos: situs inversus y rotación intestinal anormal 7 . Por motivos del presente caso, nos enfocaremos en el último.

Durante la etapa embrionaria, la rotación intestinal sucede en tres fases. En la primera, durante las semanas 5 y I0, extraembrionariamente se produce una rotación de $90^{\circ}$ en sentido antihorario, con posterior retorno del intestino medio al abdomen II. En la segunda, en la semana II, continúa la rotación de manera intraembrionaria hasta completar $270^{\circ}$. Finalmente, en la tercera etapa, se produce la fusión y el anclaje del mesenterio; el ciego desciende y el colon (ascendente y descendente) se adhiere a la pared abdominal posterior ${ }^{\text {II }}$. La rotación intestinal anormal se origina por una falla en la rotación intestinal sobre los vasos mesentéricos superiores, que ocurre entre las semanas Io y I2 de la vida fetal ${ }^{16}$. Se han descrito diferentes variaciones de esta alteración, entre ellas, la rotación inversa, así como diferentes grados de rotación irregular ${ }^{8}$.

Se cree que la rotación intestinal anormal ocurre, aproximadamente, en 0,03\% de la población y podría llegar hasta el I \%; sin embargo, su verdadera frecuencia se desconoce ${ }^{(1,9)}$. Akbulut, et al., hicieron una revisión de 95 reportes de casos de apendicitis aguda izquierda. El $60 \%$ de estos pacientes eran hombres y, entre las anomalías congénitas encontradas, la rotación intestinal anormal se reportó en el 24,2 \% frente al 69,4\% de situs inversus ${ }^{(\mathrm{I})}$. Según un estudio del Massachusetts General Hospital, el $48 \%$ de los pacientes diagnosticados con rotación intestinal anormal fueron adultos y $31 \%$ pacientes menores de un año de edad, hecho que contrastó con estudios anteriores que reportaron el diagnóstico de esta entidad hasta en el 90\% de los pacientes en su primer año de vida ${ }^{\mathrm{IO}}$.

El diagnóstico de la apendicitis aguda es primordialmente clínico, con signos y síntomas bien conocidos, además de tener en cuenta los hallazgos de imaginología, y el conocimiento y experiencia del cirujano ${ }^{17}$. De igual manera, el cuadro hemático muestra leucocitosis en más del $90 \%$ y neutrofilia en el $95 \%$ de los pacientes con esta entidad ${ }^{2}$.

Con el fin de hacer un diagnóstico oportuno mediante sencillos criterios de clasificación, Alvarado creó una escala diagnóstica para la apendicitis aguda en $1986^{18}$. Se basa en tres síntomas, tres signos y dos hallazgos de hemograma como criterios; el puntaje es de o a Io: o a 4 es negativo para apendicitis y 90 Io es apendicitis. Cuenta con una sensibilidad mayor del $80 \%$ y, en nuestro medio, se ha demostrado que es una herramienta útil en la práctica para el diagnóstico de esta entidad ${ }^{14}$.

No obstante, la apendicitis aguda en el cuadrante inferior izquierdo es poco frecuente, ocurre hasta en 0,2\% de los pacientes adultos y puede llevar a confusiones en su diagnóstico ${ }^{7}$. Por esta razón, es importante tener en cuenta los diagnósticos diferenciales del dolor abdominal en dicho cuadrante en la población adulta, como cólico renal, diverticulitis, quiste ovárico roto, epididimitis, hernia incarcerada o estrangulada, obstrucción intestinal, absceso del psoas o enteritis regional, entre otros ${ }^{19}$.

En cuanto a las imágenes diagnósticas, la ultrasonografía de abdomen es un buen apoyo en el diagnóstico de la apendicitis aguda y de la rotación intestinal anormal. Sin embargo, tiene como inconvenientes el depender del operador y brindar una limitada visualización del cuadrante inferior izquierdo por la presencia de gas en las asas intestinales ${ }^{\mathrm{I}}$.

La tomografía computadorizada (TC) es el método de elección, porque suministra información sobre la ubicación de los vasos mesentéricos (Whirpool sign), la viabilidad intestinal, la presencia de vólvulos y la asociación con otras malformaciones ${ }^{8}$. Tiene una capacidad diagnóstica adecuada para la apendicitis aguda (sensibilidad de 90 a $98 \%$ 
y especificidad de 97 a IOO\%) ${ }^{6,20,21}$. Sin embargo, la ausencia de alteraciones anatómicas en la TC no descarta por completo la rotación intestinal anormal ${ }^{12,21}$. Por otra parte, la laparoscopia es considerada el método estándar en casos de diagnóstico complejo ${ }^{\mathrm{I}}$, además de ser terapéutica, pues permite practicar en estos casos la apendicectomía ?

En el paciente que se presenta, la clínica y los exámenes de laboratorio le dieron un puntaje de 5 en la escala de Alvarado, lo cual apuntaba a una posible apendicitis. No obstante, su presentación en un lugar diferente al usual llevó a practicar estudios de imagen que evidenciaron apendicitis aguda izquierda y rotación intestinal anormal con válvula ileocecal en la fosa ilíaca izquierda.

Usualmente, en los servicios de gastroenterología y cirugía general no se tiene en cuenta una malformación congénita como diagnóstico diferencial en caso de dolor abdominal agudo en el adulto ${ }^{10,22}$. Esto ocasiona un retraso en el diagnóstico, aumenta las complicaciones operatorias y perioperatorias, genera una evolución tórpida de la enfermedad e incrementa las tasas de morbimortalidad ${ }^{23,24)}$.

En cuanto al tratamiento de la rotación intestinal anormal, algunos autores afirman que el quirúrgico con fijación del intestino es ideal para prevenir futuras complicaciones, como es el caso del vólvulo y la torsión. Estas pueden convertirse en urgencias quirúrgicas y afectar gravemente el estado de salud del paciente con complicaciones fatales ${ }^{12,25-28}$. Por otra parte, en nuestro medio se han llevado a cabo varios estudios comparando la apendicectomía abierta versus la realizada por laparoscopia, demostrando que esta última disminuye de manera significativa la morbilidad general, la tasa de infección del sitio operatorio (de 8.8 - I0.3\% con la técnica abierta vs. I.I-2.8\% con la laparoscópica), en especial la infección de la incisión del mismo y la presencia de abscesos intraabdominales, además de disminuir la estancia hospitalaria (a pesar de contar con mayor tiempo quirúrgico); por dichas razones es hoy en día el método estándar recomendado para el tratamiento de la apendicitis aguda ${ }^{29-31}$. De igual manera, la laparoscopia permite el abordaje terapéutico definitivo de la apendicitis aguda en pacientes con rotación intestinal anormal ${ }^{7}$, además de ser el método estándar para el diagnóstico en casos complejos ${ }^{\mathrm{I}}$.

Se ha encontrado asociación con diversos síndromes en el 53,2 \% de la población que presenta rotación intestinal anormal; los principales son síndrome de Down, heterotaxia y síndrome VACTERL, en orden de frecuencia ${ }^{13}$. Esta asociación ocasiona retraso para el tratamiento quirúrgico en pacientes con rotación intestinal anormal detectados al nacimiento, por riesgo de aumentar la mortalidad. No obstante, no se han evidenciado diferencias significativas de mortalidad entre la práctica temprana y la tardía del procedimiento quirúrgico para corregir la rotación intestinal anormal en neonatos ${ }^{28}$.

A pesar de la evidencia a favor de la corrección de la rotación intestinal anormal, en este caso solo se trató el cuadro agudo (apendicectomía) por decisión del cirujano, puesto que el paciente nunca había presentado síntomas asociados con su enfermedad de base. Por otro lado, se encontró una insuficiencia valvular aórtica secundaria a valvulopatía aórtica bivalva durante su seguimiento, razón por la cual se quiso buscar la posible relación entre la rotación intestinal anormal y los defectos cardiovasculares.

Los defectos cardiovasculares congénitos tienen una frecuencia de I en IOo nacidos vivos y se encuentran entre los defectos congénitos más comunes al nacimiento ${ }^{32}$. Entre ellos, la válvula aórtica bicúspide es uno de los tipos más comunes y está presente, aproximadamente, en I \% de la población ${ }^{33}$.

La información es limitada en la literatura y se describe la existencia de la rotación intestinal anormal únicamente en 2,8 a 4,I \% de los pacientes con defectos cardiovasculares congénitos ${ }^{34}$. Sin embargo, en un estudio retrospectivo en los Países Bajos, que incluyó casos encontrados entre 1980 y 2005, se encontró que los defectos cardiovasculares congénitos se presentan en el $27, \mathrm{I} \%$ de la población con rotación intestinal anormal, entre los que se encuentran defectos mayores $(48, \mathrm{I} \%)$ y menores $(5 \mathrm{I}, 9 \%)^{13}$. En este 
estudio, aunque se tienen en cuenta los defectos cardiovasculares menores, entre los pacientes de la muestra no hubo ninguno con valvulopatía aórtica bivalva, como en el presente paciente.

Actualmente, no se conocen síndromes o causas que expliquen la coexistencia de estas dos entidades. No obstante, el hecho de que en el estudio anteriormente mencionado se haya encontrado una prevalencia alta de la relación entre estas dos entidades, sugiere una causa común entre ellas ${ }^{13}$. Se han descrito alteraciones en el gen GATA6, tanto en defectos cardiovasculares congénitos como en la rotación intestinal anormal ${ }^{35}$. Reportes de casos de familias con malformaciones cardiovasculares congénitas, quienes a su vez presentaron malformaciones del eje izquierdo-derecho, mostraron defectos de lateralidad autosómicos dominantes. Algunos genes se han visto asociados con heterotaxia en humanos, como ZIC3, LEFTYA, NODAL y $A C V R 2 B$, entre otros. De igual manera, se han informado mutaciones en CRELDI y se ha visto una posible asociación con el cromosoma 6 p2 ${ }^{36}$.

\section{Conclusiones}

Es importante conocer los diversos diagnósticos diferenciales del dolor abdominal agudo en el cuadrante inferior izquierdo, para que en el momento de enfrentarse con un paciente que consulte por esta sintomatología en el servicio de urgencias, no se pasen por alto presentaciones infrecuentes de enfermedades conocidas, como la apendicitis aguda izquierda.

La rotación intestinal anormal en adultos se ha encontrado con mayor frecuencia en los últimos años, razón por la cual su conocimiento puede llevar a un diagnóstico oportuno que evitará complicaciones potencialmente mortales. Asimismo, se hace énfasis en la necesidad de conocer la relación que existe entre la rotación intestinal anormal y los defectos cardiovasculares congénitos, más específicamente con los defectos cardiovasculares menores para, de esta manera, hacer el seguimiento oportuno y prevenir complicaciones infortunadas.

\section{Referencias}

I. Akbulut S, Ulku A, Senol A, Tas M, Yagmur Y. Leftsided appendicitis: Review of 95 published cases and a case report. World J Gastroenterol. 2010;16:5598-602.

2. Chuang TJ, Chen CW, Lin HY, Hsu WH, Wang SC, Tu CC. Acute appendicitis presenting as unusual left upper quadrant pain. Iran J Radiol. 2013;I0:156-9. doi: I0.58I2/iranjradiol.6326.

3. Odabasi M, Arslan C, Abuoglu H, Gunay E, Yildiz MK, Eris C, et al. An unusual presentation of perforated appendicitis in epigastric region. Int J Surg Case Rep. 20I4;5:76-78. doi: IO.IOI6/j.ijscr.20I3.I2.005.

4. Tawk CM, Zgheib RR, Mehanna S. Unusual case of acute appendicitis with left upper quadrant abdominal pain. Int J Surg Case Rep. 2012;3:399-4OI. doi: IO.IOI6/j. ijscr.2012.05.002.

5. Birnbaum BA, Wilson SR. Appendicitis at the millennium. Radiology. 2000;215:337-48. doi: Io.II48/ radiology.215.2.rooma24337.

6. Hou SK, Chern CH, How CK, Kao WF, Chen JD, Wang LM, et al. Diagnosis of appendicitis with left lower quadrant pain. J Chin Med Assoc. 2005;68:599-603.

7. Arévalo C, Owen K, Tabares C, Bernal F, Borráez BA. Apendicitis aguda izquierda: presentación infrecuente de una enfermedad frecuente. Revisión de la literatura y reporte de un caso. Rev Colomb Cir. 2015;30:146-50.

8. Jiménez-Fuertes M, Costa-Navarro D, Zamora-Amorós C. Abdominal pain and intestinal malrotation in adults. Rev Esp Enferm Dig. 2013;105:105-6.

9. Kapfer SA, Rappold JF. Intestinal malrotation - not just the pediatric surgeon's problem. J Am Coll Surg. 2004;I99:628-35. doi: IO.IOI6/j.jamcollsurg.2004.04.024.

Io. Nehra D, Goldstein AM. Intestinal malrotation: Varied clinical presentation from infancy through adulthood. Surgery. 20II;I49:386-93. doi: Io.IOI6/j.surg.20I0.07.004.

II. Sözen S, Güzel K. Intestinal malrotation in an adult: Case report. Ulus Travma Acil Cerrahi Derg. 20I2;18:280-2. doi: I0.5505/tjtes.20I2.60973.

I2. Husberg B, Salehi K, Peters T, Gunnarsson U, Michanek $\mathrm{M}$, Nordenskjöld A, et al. Congenital intestinal malrotation in adolescent and adult patients: A I2-year clinical and radiological survey. SpringerPlus. 20I6;5:245. doi: I0.II86/s40064-0I6-I842-0.

I3. Kouwenberg M, Severijnen RSVM, Kapusta L. Congenital cardiovascular defects in children with intestinal malrotation. Pediatr Surg Int. 2008;24:257-63.

I4. Ospina JM, Barrera LF, Manrique FG. Utilidad de una escala diagnóstica en casos de apendicitis aguda. Rev Colomb Cir. 20II;26:234-4I.

15. Yang CY, Liu HY, Lin HL, Lin JN. Left-sided acute appendicitis: A pitfall in the emergency department. J Emerg Med. 20I2;43:980-2. doi: Io.IOI6/j. jemermed.20I0.II.056. 
I6. Pulgarín-Ricardo LG, Mendoza-Plata N, Ochoa-Escudero M, Donado-Gómez JH. Apendicitis aguda complicada en un paciente con malrotación intestinal. Reporte de caso. Iatreia. 2009;22:77-9.

I7. Herrera-Moncada IC, Zuluaga-Restrepo JD, Meza M. Apendicitis aguda en situs inversus totalis: reporte de un caso. Rev CES Med. 20I2;26:59-64.

I8. Alvarado A. A practical score for the early diagnosis of acute appendicitis. Ann Emerg Med. 1986;15:557-64.

19. Akbulut S, Caliskan A, Ekin A, Yagmur Y. Left-sided acute appendicitis with situs inversus totalis: Review of 63 published cases and report of two cases. J Gastrointest Surg. 20IO;I4:I422-8. doi: IO.IOO7/sII605-OIO-I2IO-2.

20. Perera WR, Hennessy OF. Clinical Images. An unusual case of appendicitis. Am J Surg. 20I0;199:e79-8I. doi: IO.IoI6/j.amjsurg.2009.08.047.

2I. Mun S, Ernst RD, Chen K, Oto A, Shah S, Mileski WJ. Rapid CT diagnosis of acute appendicitis with IV contrast material. Emerg Radiol. 2006;I2:99-IO2. doi: I0.IO07/siol40-005-0456-6.

22. Nagdeve NG, Qureshi AM, Bhingare PD, Shinde SK. Malrotation beyond infancy. J Pediatr Surg. 2012;47:2026-32. doi: I0.IOI6/J.jpedsurg.2012.06.013.

23. Welte FJ, Grosso M. Left-sided appendicitis in patient with congenital gastrointestinal malrotation: A case report. J Med Case Reports. 2007;I:92. doi: IO.II86/I752I947-I-92.

24. Au ACY, Syed A, Bradpiece HA. A rare case of intestinal malrotation presenting as appendicitis in late adulthood. J Surg Case Rep. 20I0;20I0:3. doi: I0.IO93/ jscr/20Io.8.3.

25. Chang J, Brueckner M, Touloukian RJ. Intestinal rotation and fixation abnormalities in heterotaxia: Early detection and management. J Pediatr Surg. 1993;28:128I5. doi: I0.IOI6/Soo22-3468(05)80313-6.

26. Tashjian DB, Weeks B, Brueckner M, Touloukian RJ. Outcomes after a Ladd procedure for intestinal malrotation with heterotaxia. J Pediatr Surg. 2007;42:528-3I.

27. Prasil P, Flageole H, Shaw KS, Nguyen LT, Youssef S, Laberge JM. Should malrotation in children be treated differently according to age? J Pediatr Surg. 2000;35: 756-8.
28. Sulkowski JP, Cooper JN, Duggan EM, Balci O, Anandalwar S, Blakely ML, et al. Early versus delayed surgical correction of malrotation in children with critical congenital heart disease. J Pediatr Surg. 2015;50:86-9I. doi: Io.IoI6/j.jpedsurg.20I4.I0.0o6.

29. Mosquera M, Kadamani A, Pacheco M, Villarreal R, Ayala JC, Fajardo LP, et al. Apendicectomía laparoscópica versus abierta: comparables. Rev Colomb Cir. 20I2;27:I2I-I28.

30. Onieva FG, Roldán S, Domínguez JR, Montero JP, Galnares A, Peralta J. Abordaje laparoscópico frente a enfoque clásico en el tratamiento de la appendicitis aguda. Rev Colomb Cir. 20I7;32:26-3I. doi: I0.30944/20II7582.4

3I. Toro JP, Barrera OJ, Morales CH. Superioridad clínica de la apendicectomía laparoscópica sobre la técnica abierta: ¿adopción lenta de un Nuevo estándar de tratamiento?. Rev Colomb Cir. 20I7;32:32-39. doi: I0.30944/20II7582.5.

32. Hoffmann JIE, Kaplan S. The incidence of congenital heart Disease. J Am Coll Cardiol. 2002;39:1890-1900.

33. Braverman AC. The bicuspid aortic valve and associated aortic disease. En: Otto CM, Bonow RO, editores. Valvular Heart Disease. Fourth edition. Philadelphia: Saunders-Elsevier; 2013. p. I79.

34. Pradat P, Francannet C, Harris JA, Robert E. The epidemiology of cardiovascular defects, part I: A study based on data from three large registries of congenital malformations. Pediatr Cardiol. 2003;24:I95-22I. doi: I0.IOO7/s00246-002-940I-6.

35. Yu L, Bennett JT, Wynn J, Carvill GL, Cheung YH, Shen $\mathrm{Y}$, et al. Whole exome sequencing identified de novo mutations in GATA6 associated with congenital diaphragmatic hernia. J Med Genet. 2014;51:197-202. doi: Io.II36/jmedgenet-20I3-IOI989.

36. Wessels MW, De Graaf BM, Cohen-Overbeek TE, Spitaels SE, De Groot-de Laat LE, Ten Cate FJ, et al. A new syndrome with noncompaction cardiomyopathy, bradycardia, pulmonary stenosis, atrial septal defect and heterotaxy with suggestive linkage to chromosome 6p. Hum Genet. 2008;I22:595-603. doi: I0.I007/s00439-0070436-x. 